\title{
Reducing the beam impedance of the kicker at the 3-GeV rapid cycling synchrotron of the Japan Proton Accelerator Research Complex
}

\author{
Yoshihiro Shobuda, Yong Ho Chin, Naoki Hayashi, Yoshiro Irie, Tomohiro Takayanagi, \\ Tomohito Togashi, Takeshi Toyama, Kazami Yamamoto, and Masanobu Yamamoto \\ J-PARC Center, JAEA\&KEK, 2-4 Shirakata, Tokaimura, Nakagun, Ibaraki 319-1195, Japan
}

(Received 25 December 2017; published 11 June 2018)

\begin{abstract}
The present four-terminal kicker at the rapid cycling synchrotron (RCS) at the Japan Proton Accelerator Research Complex has the power-saving benefit that it allows beam extraction by doubling the excitation currents with two shorted ends. In this configuration, two terminals of the kicker are connected to the pulseforming line while the other two are terminated in a short circuit. On the other hand, beam instabilities are excited in the RCS by the kicker beam impedances, which result from the short-circuit termination of the kicker. In this paper, we describe a scheme to reduce the beam impedance of the kicker using diodes (nonlinear devices), while retaining the benefit of the doubled kicker excitation currents. We employ a simulation technique to determine the beam impedance of the kicker, even when such nonlinear devices and long cables are included. The characteristic of beam impedance measured using the accelerated beams is well explained by that obtained from the simulation.
\end{abstract}

DOI: 10.1103/PhysRevAccelBeams.21.061003

\section{INTRODUCTION}

The 3-GeV rapid cycling synchrotron (RCS) at the Japan Proton Accelerator Research Complex (J-PARC) [1] aims to achieve a megawatt-class beam. When two bunched beams, each containing $4.15 \times 10^{13}$ particles per bunch, are accelerated from $400 \mathrm{MeV}$ to $3 \mathrm{GeV}$ at a repetition rate of $25 \mathrm{~Hz}$, a 1-MW beam can be formed at the RCS.

Beam instability [2] is the main issue that limits the generation of a high-intensity beam at the RCS [3]. We have already observed such instabilities in beams with over $300 \mathrm{~kW}$-equivalent power and full chromaticity correction. We have also demonstrated that the kicker resonant beam impedance, resulting from the short-circuit termination of the kicker, excites beam instability: this was demonstrated by terminating the ends of the kicker cables with resistors to suppress the beam-impedance resonances [2-4]. Unfortunately, since the impedance matching between the pulse-forming line (PFL) and the kicker cables does not hold due to the resistor insertion, the kicker magnet current decreases to two thirds of the nominal current, which is not sufficient for beam extraction.

On the other hand, we have investigated and clarified a mechanism for suppressing space-charge effects on beam instability in a space-charge dominated machine like the

Published by the American Physical Society under the terms of the Creative Commons Attribution 4.0 International license. Further distribution of this work must maintain attribution to the author(s) and the published article's title, journal citation, and DOI.
RCS, both in theoretical and simulation manners [5,6]. By making maximum use of space-charge damping on the beam instability, we have successfully accelerated a 1-MWequivalent beam by enlarging the transverse beam emittance, manipulating the chromaticity correction pattern, and optimizing the tune-tracking pattern during the acceleration period [5].

The RCS provides proton beams downstream to both the Material and Life science Facility (MLF) and the Main Ring (MR) [1]. By switching beam targets, the RCS can manipulate the beam parameters for the MLF or the MR.

The standard transverse emittance for the MLF is relatively large (e.g. $200 \pi \mathrm{mm}$-mrad). The larger emittance can easily stabilize a high-intensity beam, even for a 1-MW beam [5]. On the other hand, for the MR, the transverse emittance can be less than $50 \pi \mathrm{mm}$-mrad. The smaller transverse emittance broadens the transverse tune spreads due to space-charge effects, restricting the variable tune-tracking pattern during the acceleration period. Consequently, it is more difficult to suppress instability for a beam with a smaller transverse emittance than for one with a larger transverse emittance.

The kicker at the RCS is a transmission-line type combined with two ferrite cores [7]. The kicker has four terminals, two of which are connected to the power supply through 130-m-long coaxial cables, and the other two being terminated in a short circuit. These terminal conditions are beneficial for the extraction of beams, because they ensure doubled excitation currents by the shorted ends.

A direct solution for suppressing the beam instability is to reduce the beam impedance of the kicker (the source of 
the beam instability). A straightforward way to accomplish this is to connect matched resistors to the kicker terminals to absorb beam-induced currents [8]. However, this scheme eliminates the merit of the doubled excitation currents available from the present kicker.

In this paper, we discuss a solution for suppressing the beam impedance of the kicker while retaining the performance of a kicker with short-circuit terminations. In Sec. II, we describe the present kicker magnet and the beam impedance. In Sec. III, we propose a new method using a diode to reduce the beam impedance of the kicker while retaining the benefit of the doubled excitation currents. In Sec. IV, we describe a simulation technique for determining the beam impedance of the kicker, including the 130-mlong coaxial cables and the nonlinear device (diode). In Sec. V, we present measurements of the reduced beam impedance of the kicker using high-intensity beams at the RCS. The paper is summarized in Sec. VI.

\section{PRESENT RCS KICKER AT J-PARC}

\section{A. The parameters of the kicker}

As shown in Fig. 1, the kicker at the $3-\mathrm{GeV}$ RCS at J-PARC consists of a magnet and two pulse-forming lines (PFLs). Aluminum (Al) coils are embedded in the kicker magnet, which is a window-frame transmission-line type consisting of two ferrite cores, each of $9.8 \Omega$ characteristic impedance (the mutual coupling between both cores is negligible at the RCS kicker [9]). The magnet has four terminals. Two terminals are connected to 130-m-long coaxial cables, while the other two are terminated by shorting plates. This creates doubled excitation currents due to the shorted ends by inverting the voltage wave front, when the system is pulsed to extract beams from the RCS.

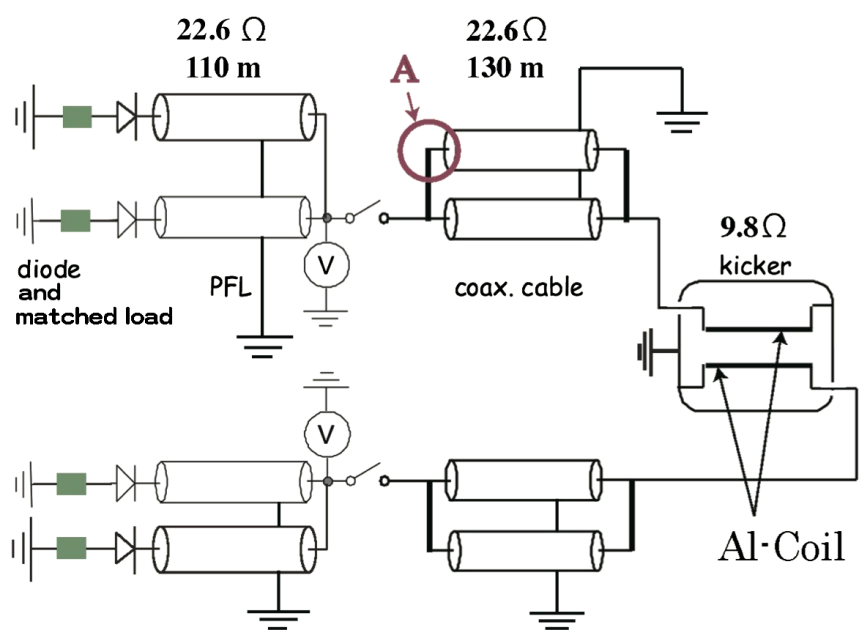

FIG. 1. A schematic overview of the kicker magnet, highlighting the $\mathrm{Al}$ coil and the pulse-forming lines (PFLs), in terms of electric circuit theory $[7,8]$. The location "A" shows the current monitoring point of Fig. 7.
The high power switch, between PFLs and coaxial cables, is only closed to extract beam: hence, these ends of the coaxial cables connecting to the PFLs are completely open during the acceleration of a beam.

The characteristic impedance $Z_{\text {cable }}$ of the power cable and the wave propagation speed $v_{\text {cable }}$ in the power cable are about $22.6 \Omega$ and $0.57 \times c \mathrm{~m} / \mathrm{s}$, respectively, where $c$ is the velocity of light. The cable length is $130 \mathrm{~m}$. Two coaxial cables are connected in parallel to one terminal of the kicker, to match the impedance of the kicker magnet as well as possible. The combined resistivity $R_{\text {cable }}$ of the inner and outer conductors in the cable, and the conductance $G$ of the insulator between the conductors, are given by

$$
R_{\text {cable }}=\frac{1}{2}\left(\frac{1}{a_{\text {cable }}}+\frac{1}{b_{\text {cable }}}\right) \sqrt{\frac{Z_{0} f}{c \pi \sigma_{c}}},
$$

$$
G=2 \pi f \mathcal{C}_{\text {cable }} \tan \delta,
$$

where $\mathcal{C}_{\text {cable }}=1 /\left(v_{\text {cable }} Z_{\text {cable }}\right)$ is the capacitance of cable per unit length, $f$ is the frequency, $Z_{0}=120 \pi \Omega$ is the impedance of free space, $a_{\text {cable }}=9.1 \mathrm{~mm}$ and $b_{\text {cable }}=$ $15 \mathrm{~mm}$ are the radii of the inner and the outer conductors of the cable, respectively, $\sigma_{c}=4.48 \times 10^{7} \mathrm{~S} / \mathrm{m}$ is the conductivity of the conductors, and $\tan \delta=0.018$ is $\tan -\delta$ of the insulator between the conductors.

Three types of kicker (the S-type, M-type, and L-type), which are distinguished by their gap height $2 b$, are installed in the RCS. In this paper, unless specified otherwise, we analyze only the L-type kicker $(2 b=199 \mathrm{~mm})$. A photograph and a schematic illustration of the kicker are shown in Fig. 2. The kicker magnet consists of aluminum alloy components (the high voltage plates, the flux break, the grounding plates, and the vessel) and ferrite (Ni-Zn ferrite PE14, TDK Ltd. [10]) components. The aluminum coils and the flux break penetrate the kicker in the longitudinal direction. The aluminum coils and the high voltage plates are electrically connected, while the flux break and the grounding plates are done to the vessel holding them. The ferrite is sandwiched by the high voltage plates. The relative permeability $\mu^{\prime}$ of the ferrite is approximated as

$$
\mu^{\prime}=7.0+\frac{800.0}{1.0+j 2 \pi f \tau}
$$

where $j$ is imaginary unit, and the relaxation time $\tau=1.59155 \times 10^{-7} \mathrm{~s}$. The relative dielectric constant $\epsilon^{\prime}$ of the ferrite is equal to 6.2, regardless of the frequency.

\section{B. The beam impedance of the present kicker}

The beam impedances of the kicker have been thoroughly investigated in Refs. [8,11]. Notice that there is no 

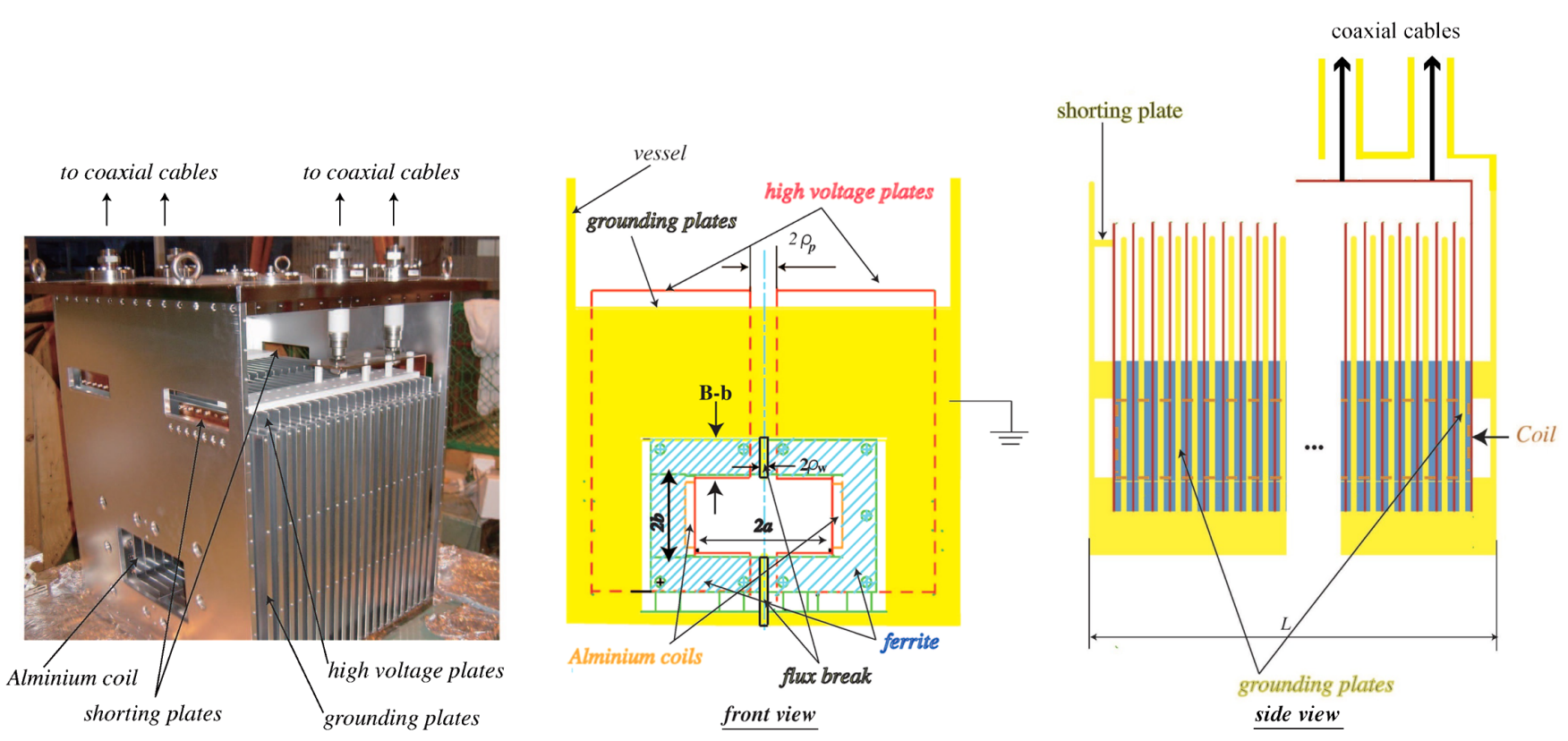

FIG. 2. A photograph (left) and a schematic illustration (middle/right) of the RCS kicker at J-PARC.

beam screen at the RCS kicker, as shown in Fig. 2. In Ref. [8], theoretical results and measurements of the longitudinal beam impedance $Z_{L}$ and of the horizontal beam impedance $Z_{x}$ are compared for beams with Lorentz$\beta=0.5453,0.75$, and 0.9 , under the condition that the ends of the cables are terminated with matched resistors. We have demonstrated that $Z_{L}$ does not significantly depend on the Lorentz- $\beta$, while $Z_{x}$ is roughly proportional to Lorentz$\beta$ at low frequency $[8,12]$.

We performed a consistency check between the theoretical results and the measurements for the prototype kicker with the ends of the cables completely open, using a standard wire method $[8,13]$. We also performed a check for the beam impedance of the L-type kicker, using a nonrelativistic beam with Lorentz- $\beta=0.5453$ [8].

We have established the theory of the beam impedance of the kicker at the RCS [8]. Figure 3 shows the theoretical results for the beam impedance of the L-type kicker with a relativistic beam, as calculated in the Appendix for infinite terminal impedance $R_{T}$ (i.e., for an open terminal). Note that dispersion due to the resistivity of the cables is included in the results. The spiky structures in Fig. 3 correspond to beam-impedance resonances of the kickercables system.

Previously, measurements of longitudinal beam impedances were done, concerning the influence of coaxial cable
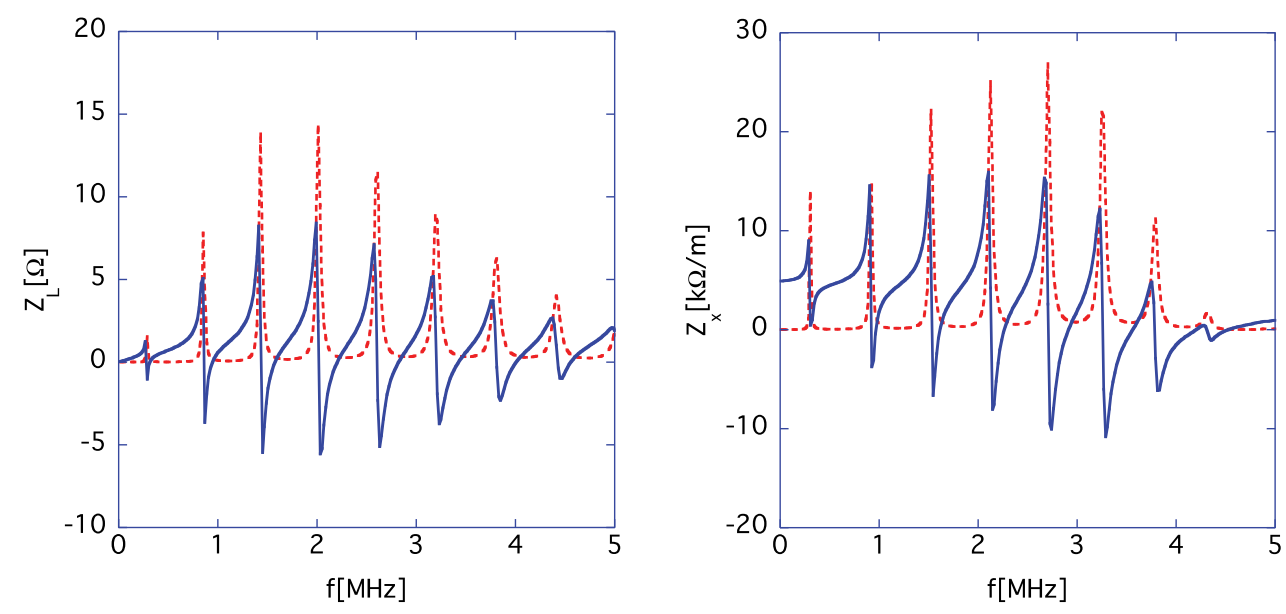

FIG. 3. Theoretical results for the longitudinal (left) and horizontal (right) beam impedances for a relativistic beam, when the cable terminals are open. The red dashed and blue solid lines show the real and imaginary parts of the beam impedances, respectively. 


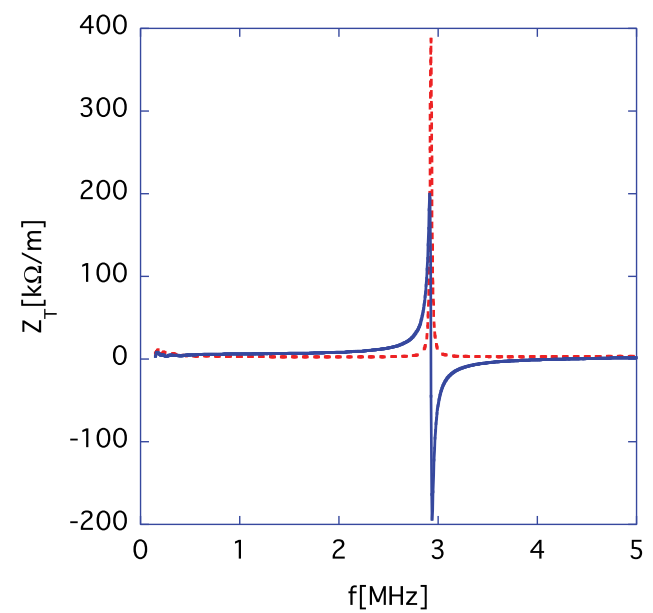

FIG. 4. Measurements of the horizontal beam impedances, where all cables are simply detached from the kicker. The red dashed and blue solid lines show the real and imaginary parts of the impedance.

connected to a short-circuited kicker magnet at TRIUMF [14]. The study aims at reducing the beam impedance with a saturating inductor, which has a high impedance for a small magnitude of currents (excited by a beam). The electric device (saturating inductor) effectively terminates the cable as an open circuit, when it is inserted between the magnet end and the corresponding cable terminal. The device successfully eliminated the beam-impedance resonances due to the coaxial cables, while the maximum value of the impedance remained the same order of magnitude (refer to Fig. 2 in Ref. [14]).

In order to discuss whether the same impedance reduction scheme can be applied to the RCS kicker, we present measurement results of the horizontal beam impedance of the RCS prototype kicker, from which all cables are detached. Following the scheme explained in Refs. [8,15], the horizontal beam impedance of the kicker with two shorted and two open terminals has been measured by making a wire loop inside the kicker aperture and measuring the reflection coefficients $S_{d d 11}$ for the differential mode with a network analyzer.

Figure 4 represents the measurement, which demonstrates the huge impedance enhancement at the J-PARC RCS kicker, though the resonance structure diminishes by detaching the cables from the kicker magnet. That is why we have to develop a new scheme to reduce the beam impedance of the kicker at the RCS at the J-PARC.

\section{A SCHEME TO REDUCE THE BEAM IMPEDANCE OF THE KICKER}

The spiky structures in Fig. 3 are created by reflection of beam-induced currents in the cables. We have already found that the beam impedance of the kicker can be reduced by attaching resistors at the ends of power cables

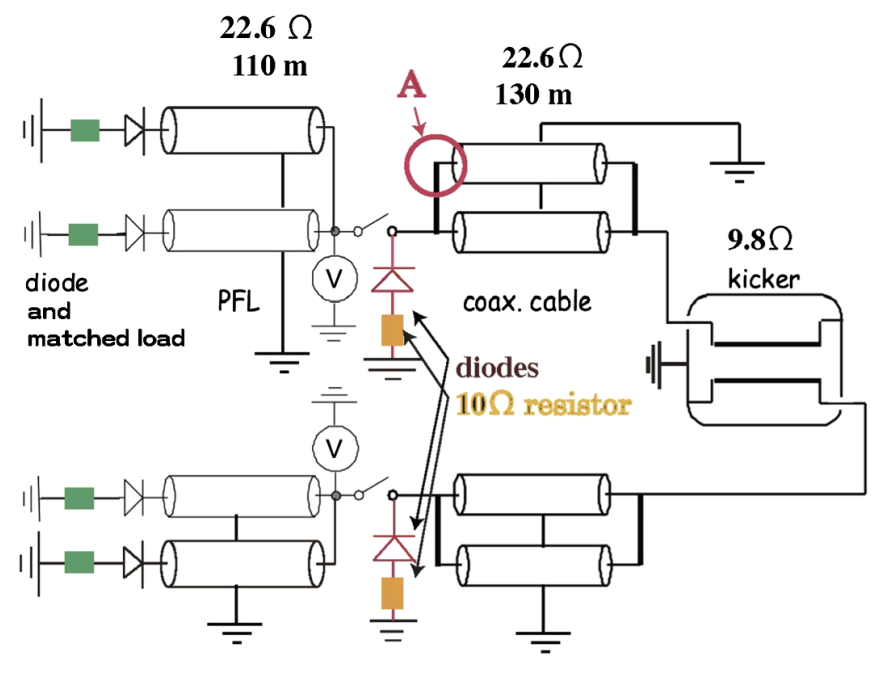

FIG. 5. A schematic illustration of the modified kicker in terms of electric circuit theory $[7,8]$. The location "A" shows the current monitoring point of Fig. 7.

$[3,4]$. However, if we simply adopt this scheme for the present kicker, the merit of the doubled excitation currents is eliminated, due to the attached resistors.

Instead, let us consider the different scheme shown in Fig. 5, in which a diode and resistor are inserted at the switch end of the coaxial cables. The diodes isolate the resistors from the switch cathode, but the resistors can still be "seen" electrically by the beam. In other words, the diode must have a sufficiently high reverse voltage $V_{R}$ to block the pulse voltage from the PFLs and a low forward voltage drop $V_{F}$ so that the lower amplitude beam-induced voltage pulses can be affected by the resistors.

At the RCS, $V_{R}$ must be $40 \mathrm{kV}$, while $V_{F}$ should be $50 \mathrm{~V}$ or lower. The (diffusion junction silicon) diode we used was specifically designed and developed by ORIGIN [16], based on the calculation of SPICE (simulation program with integrated circuit emphasis). A picture of the diode is shown in Fig. 6. The diode is composed of four parallel branches, each branch contains thirteen modules in series. Table I shows SPICE parameters for one module.

Figure 7 represents a measurement result of the current pulse rising edge provided by PFL at position A in Figs. 1 and 5. The red dotted and the blue solid lines show the results with and without diode and $10 \Omega$ resistor, respectively. The damped oscillation can be identified on the red dot line. The phenomenon is due to the present measurement setup, because the calculation of SPICE shows that the pulse rise time cannot be affected by the zero-bias junction (parasitic) capacitance solely [17]. In this measurement setup, the diodes and resistors are set inside the "container" (see Fig. 6), connected to the end of 130-m long coaxial cables through " $3.1-\mathrm{m}$ long $20 \Omega$ coaxial cable." The container and the 3.1-m long cable may create significant additional inductances and capacitances and 

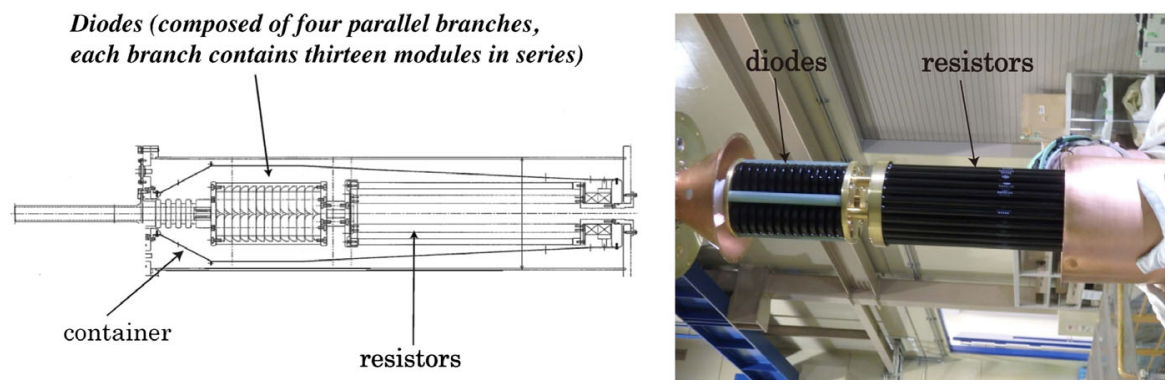

FIG. 6. A detailed schematic (left) of one diode with a $10 \Omega$ resistor from Fig. 5 and its photo (right).

deform the pulse current waveform. Since three-dimensional simulation with the CST code [18] well reproduces the measurements [17], we conclude that the impedances of the container and of the 3.1-m long coaxial cable significantly contribute to the damped oscillation on the red dotted line. Practically, the oscillation can be eliminated by shortening the cable length from 3.1 to $1 \mathrm{~m}$ or less, according to some simulation studies [17].
The voltage-current (V-I) characteristic of the module is represented in the bottom figures of Table I, which is derived from the diode module circuit with resistive load by using the CST code. The diode module dynamic resistance at $2.5 \mathrm{~V}$ forward bias and that at $12 \mathrm{~V}$ forward bias are about $2.5 \Omega$ and $1.0 \Omega$, respectively. Hence, when the voltage over the forward-biased diode increases, the current increases even faster leading to lower dynamic resistance.

TABLE I. SPICE parameters for one module, whose V-I curve (left) from -3 to $3 \mathrm{~V}$ and that (right) from 11.5 to $12.5 \mathrm{~V}$ are represented in the bottom figures. One diode shown in Fig. 5 consists of four parallel branches, each branch contains thirteen modules in series.

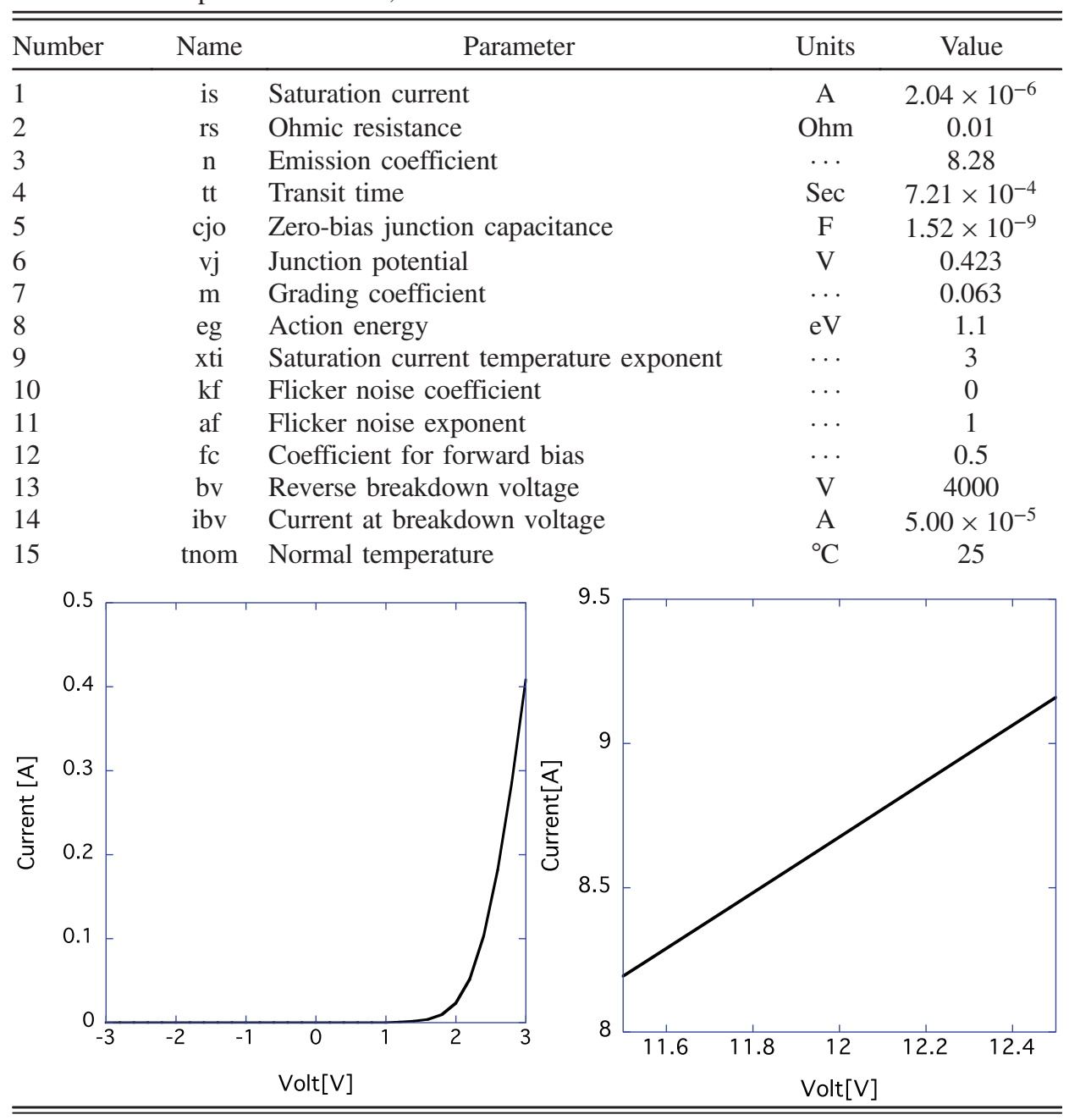




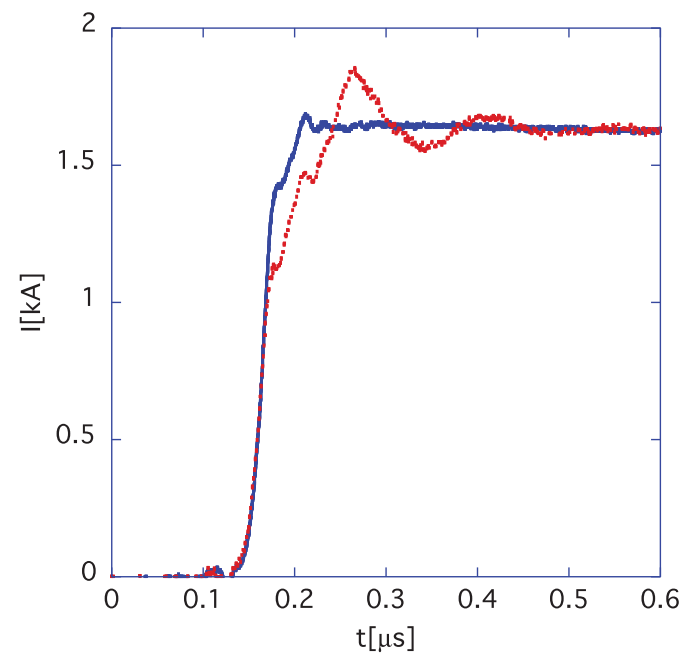

FIG. 7. Measurements of rising edge of the pulse current provided by PFL at the position A in Figs. 1 and 5. The red dotted and the blue solid lines show the results with and without diode and $10 \Omega$ resistor, respectively.

On the other hand, a beam can excite the backward voltage as well as the forward voltage on the diode. Moreover, it is possible that the parasitic capacitance of the diode deforms the beam-induced currents, while it plays a role in the resonance suppression.

Nevertheless, since the diode violates the perfect impedance matching, it may cause a significant reduction of the beam-impedance suppression. It is obvious that if there is perfect impedance matching between the cable and the load, all energy of the cable is absorbed without reflection, and the resonances will be suppressed. In other words, if impedance matching is violated, there will be reflection, causing the resonances in the impedance. In this sense, the effect of the diode on the beam impedance is not obvious.

In the following section, we show how the resonant beam impedance of the modified kicker system is reduced by the resistors, which are insulated by the nonlinear devices (the diode).

\section{EVALUATION OF THE BEAM IMPEDANCE OF THE MODIFIED KICKER IN A SIMULATION}

Since the modified kicker shown in Fig. 5 includes a diode (a nonlinear device), the wake function must first be evaluated in the time domain [2]. We then convert it into an impedance in the frequency domain.

A numerical simulation is one possible scheme for evaluating the beam impedance in three dimensions. The WAKe Solver at CST STUdio [18] can simulate the beam impedance by letting a beam pass through the kicker. However, since the kicker magnet is connected with 130m-long coaxial cables, excessive memory and CPU time are necessary to secure a sufficient mesh size, which makes the simulation unrealistic.

On the other hand, the Microwave Solver at CST STUDIO can combine a three-dimensional simulation with an electric circuit. In this way, we can deal effectively with both the 130-m-long coaxial cables and the diode represented by SPICE parameters using the electric circuit solver of the code CST STUDIO SuITE 2017. However, up to now, the scheme has not incorporated a "beam" into the simulation.

Here, we introduce an experimental aspect into the simulation [19]. A standard way to measure the beam impedance is to stretch wires (instead of passing a beam) inside the kicker and measure the scattering matrix [13]. If we assume that this measurement scheme (the wire method) reproduces the beam impedance of the kicker when excited by a beam, we can calculate the beam impedance, even if the kicker is connected to 130-m-long coaxial cables and is equipped with a nonlinear device, (i.e., the diode). Note that we have performed a consistency check between the theoretical results and the measurements for the prototype kicker with the ends of the cables completely open, using the wire method, as written in Sec. II B [8].

A schematic illustration of the wire method is shown in Fig. 8. The impedances are defined by the integration of field distortions due to a device under test (the kicker) from
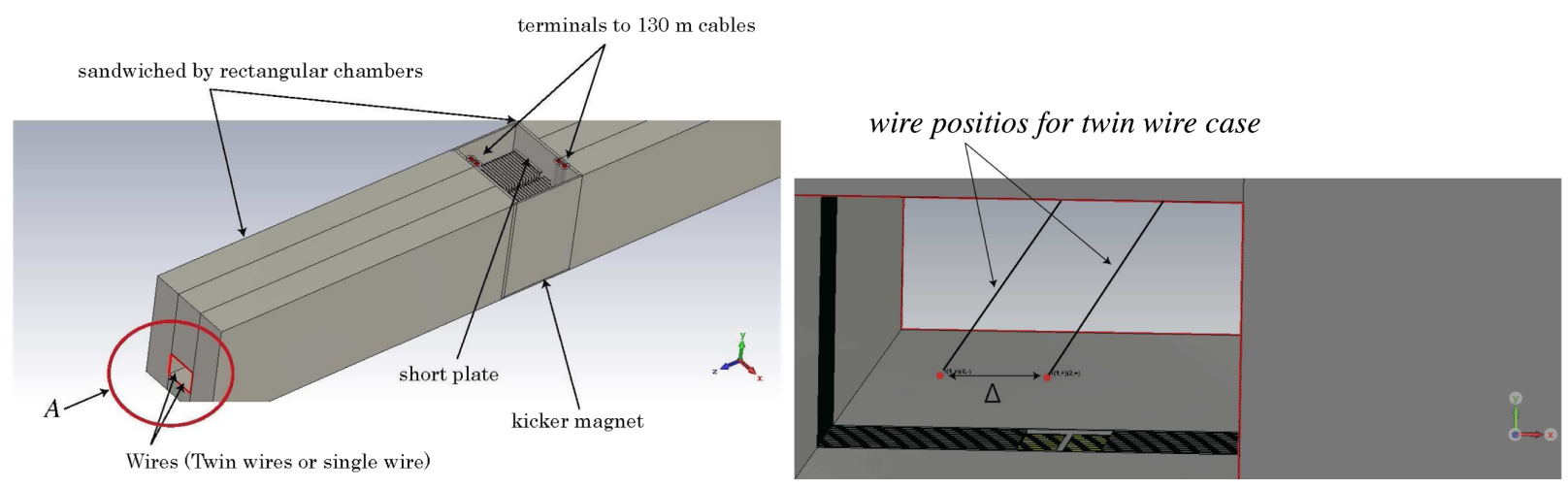

FIG. 8. Simulation setup (left) for measurements of the longitudinal or horizontal beam impedances using the wire method. The scaled up figure of area $\mathrm{A}$ in the left picture is shown in the right picture. The wire radius is $\rho_{w}=80 \mu \mathrm{m}$ and the twin-wire separation is $\Delta=60 \mathrm{~mm}$. For the measurements of the longitudinal beam impedance, the single wire is positioned at the center of the chamber. 
minus infinity to plus infinity in the longitudinal direction. Since the RCS kicker aperture is rectangular, two perfectly conducting rectangular chambers (the aperture size is identical to the aperture size of the kicker) sandwich the kicker in order to precisely simulate the distortions in this setup. As shown in Fig. 2, the kicker magnet is set inside the grounded vessel, which is electrically connected to the perfectly conducting rectangular chambers.

Off-line measurements of the longitudinal and horizontal beam impedances of the kicker are typically done by stretching single or twin wires inside the kicker sandwiched between the chambers. The terminals to the network analyzer, as well as those to the coaxial cables designated at the electric circuit part, are represented by waveguide ports at the three-dimensional part of the CST code. By connecting the entrance and the exit (denoted by the waveguide port) of the kicker with the ports of the network analyzer, we can measure the scattering matrices. We term the entrance "terminal one" and the exit "terminal two," respectively. The measured scattering matrices are converted into beam impedances by using the standard log formulas [13]:

$$
\begin{gathered}
Z_{L}=-2 Z_{c c} \log \frac{S_{c c 21}^{\mathrm{DUT}}}{S_{c c 21}^{(\mathrm{ref})}}, \\
Z_{x}=-\frac{2 c Z_{d d}}{\omega \Delta^{2}} \log \frac{S_{d d 21}^{\mathrm{DUT}}}{S_{d d 21}^{(\mathrm{ref})}},
\end{gathered}
$$

where $\Delta$ is the separation between the twin wires, $Z_{c c}$ and $Z_{d d}$ are the characteristic impedances for the common and the differential modes, respectively; $S_{c c 21}^{\mathrm{DUT}}$ and $S_{d d 21}^{\mathrm{DUT}}$ are the transmission coefficients for the kicker (DUT), and $S_{c c 21}^{(\mathrm{ref})}$ and $S_{d d 21}^{(\mathrm{ref})}$ are those for the perfectly conducting reference chamber, respectively. The subscripts $c c$ and $d d$ denote the common and the differential modes, respectively. The characteristic impedances are numerically calculated by CST code as well.

The attenuation parameter $\alpha \mathrm{dB} / \mathrm{m}$ of the cable is calculated as [20]

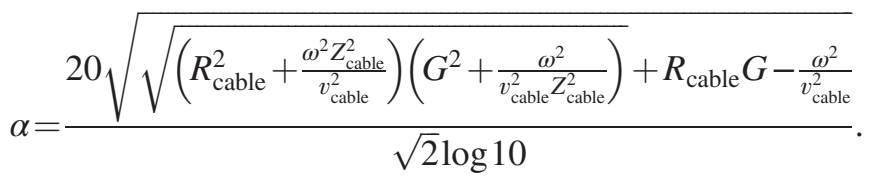

As shown in Fig. 9, though the parameter $\alpha$ has frequency dependence, only the "constant attenuation parameter" can be designated in the CST code. Considering the results of Fig. 9 as an initial step to specify the attenuation parameter, after some trial and error from simulations, we find that the overall behavior of the (analytical) results for the longitudinal and horizontal beam impedances shown in

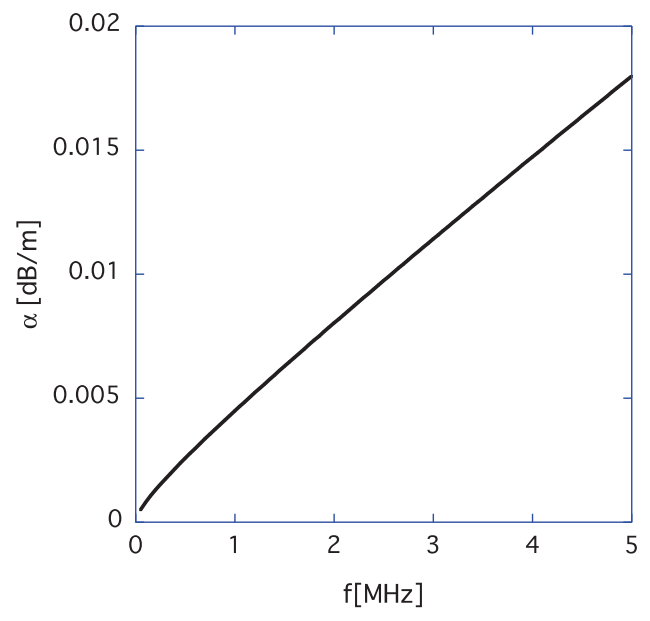

FIG. 9. The frequency dependence of attenuation parameter $\alpha$.

Fig. 3 can be well reproduced simultaneously by choosing $0.015 \mathrm{~dB} / \mathrm{m}$ and $20 \Omega$ as the attenuation and the impedance parameters of the cable in the CST code.

The simulation results for the present kicker (with open cable terminals) obtained with the MicroWAVE SOLVER of CST are shown in Fig. 10, where the wire radius is $\rho_{w}=80 \mu \mathrm{m}$ and the twin-wire separation is $\Delta=60 \mathrm{~mm}$. Here we assume the wires are perfectly conductive for both the kicker and the reference chamber, to avoid generating excessive mesh numbers in the wires $[8,11]$. The imaginary part of the horizontal beam impedance is a bit increased, because we cannot presently incorporate the detailed dispersion effect of the cable resistivity into the CST code.

From this starting point, let us next investigate how the beam impedances are reduced by connecting the diodes and resistors to the ends of the cables. This is different from conventional cases. In the typical analysis, where only linear electric devices are included in the device under test, the principle of superposition is assumed. Accordingly, a simulation is done by sweeping the frequency of a sinusoidal signal with small amplitude and single frequency. However, the beam impedance now depends on the beam intensity, due to the nonlinearity of the device. Consequently, we must run the time domain solver rather than frequency domain solver during the simulation.

As shown in the upper-left figure of Fig. 11, we detach terminals one and two from the network analyzer. We then connect terminal one to the pulse generator, while terminal two is connected to ground through a matched resistor, i.e., the resistance is equal to the characteristic impedance $Z_{c c}$ for the common mode, and $Z_{d d}$ for the differential one. The choice of the matched resistance depends whether single wire or twin wires are stretched in the simulation setup (the upper-left figure of Fig. 11 shows a simulation setup only for the single wire measurements, for simplicity). As shown in the left figure of Fig. 11, waveguide ports 3, 4, 5, and 6 of the 

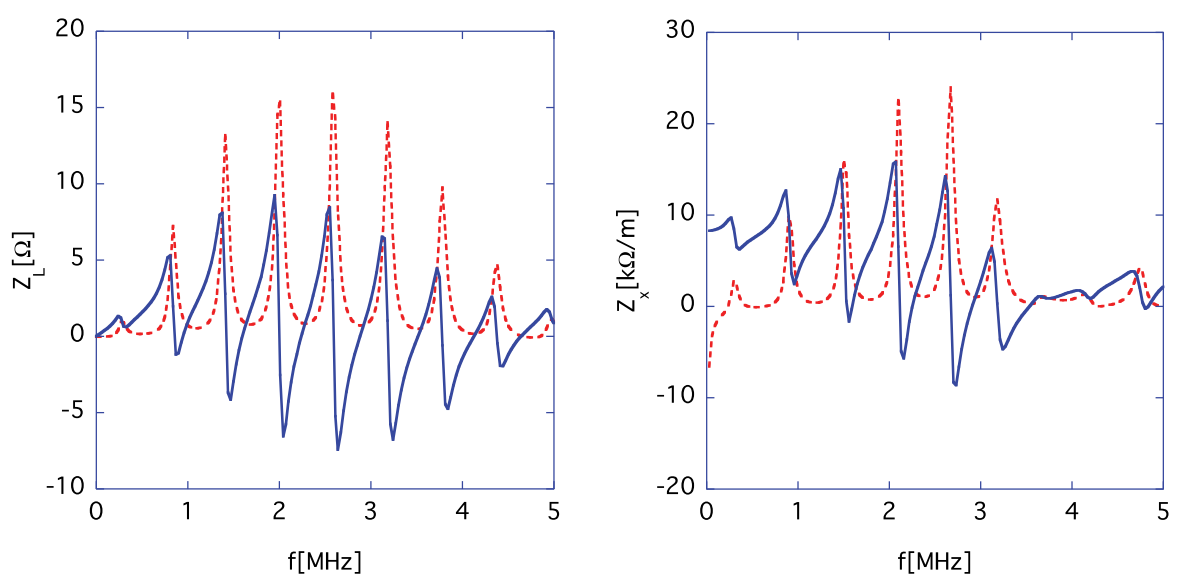

FIG. 10. Simulation results for the longitudinal and horizontal beam impedances, where the wire radius is $\rho_{w}=80 \mu \mathrm{m}$ and the twinwire separation is $\Delta=60 \mathrm{~mm}$. The red dashed and blue solid lines show the real and imaginary parts of the beam impedances, respectively.

kicker magnet are connected to the coaxial cables. All ends of the cables are terminated by the diodes and resistors at the electric circuit part, as shown in the right part of Fig. 11.

Let a current flow from the pulse generator simulating a beam pulse, while the currents and voltages at terminals one and two are monitored in the time domain. By substituting the Fourier-transformed currents and voltages into

$$
S_{c c(d d) 21}=-\frac{2 Z_{c c(d d)} \tilde{I}_{2}}{\tilde{V}_{1}+Z_{c c(d d)} \tilde{I}_{1}},
$$

we obtain the scattering matrix $S_{c c(d d) 21}$ for the given pulsed current, where $\tilde{I}_{1}$ and $\tilde{V}_{1}$ are the Fourier-transformed current and voltage at terminal one, respectively; $\tilde{I}_{2}$ is the

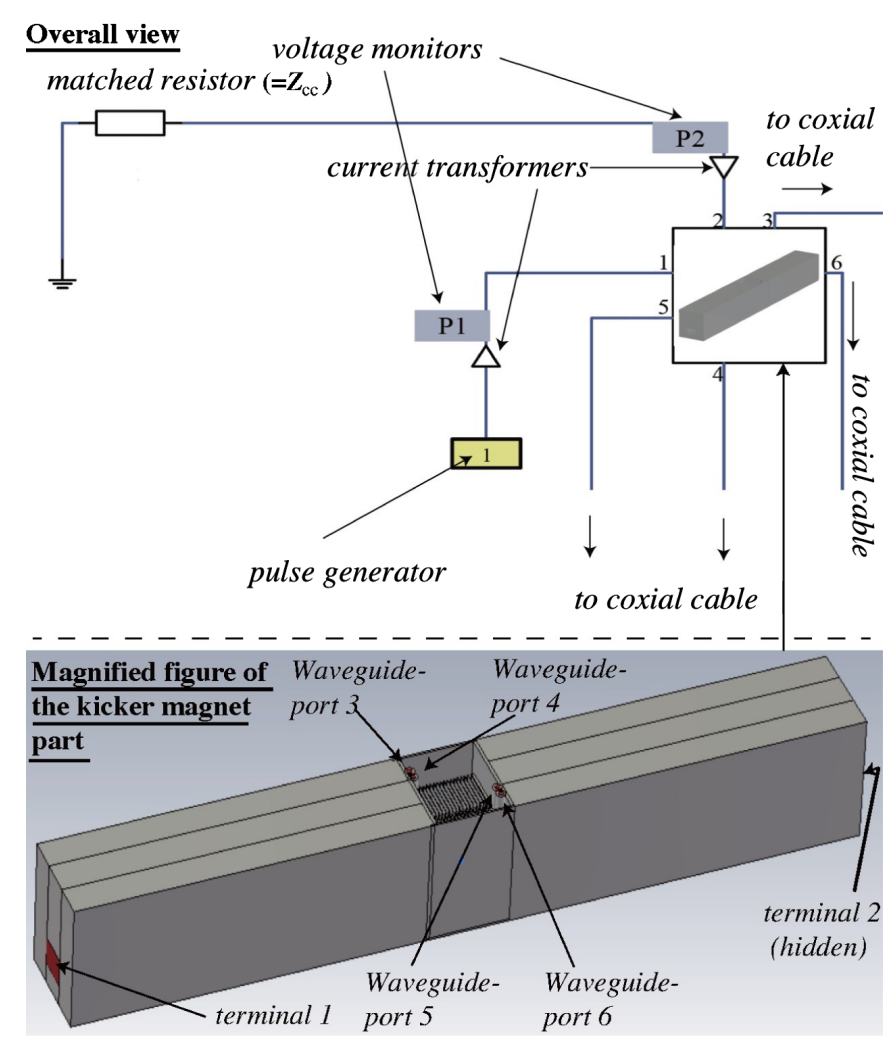

Cables, diodes and resistors parts

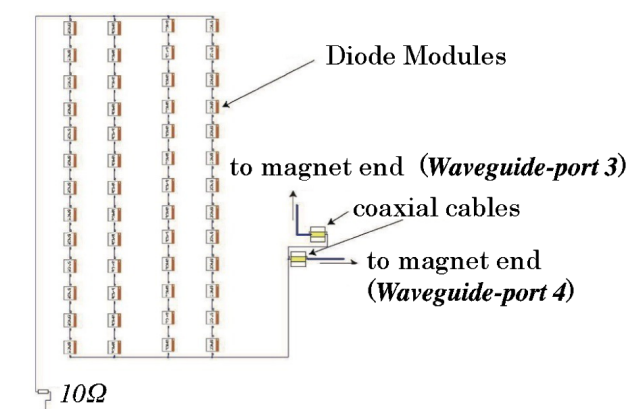

$910 \Omega$

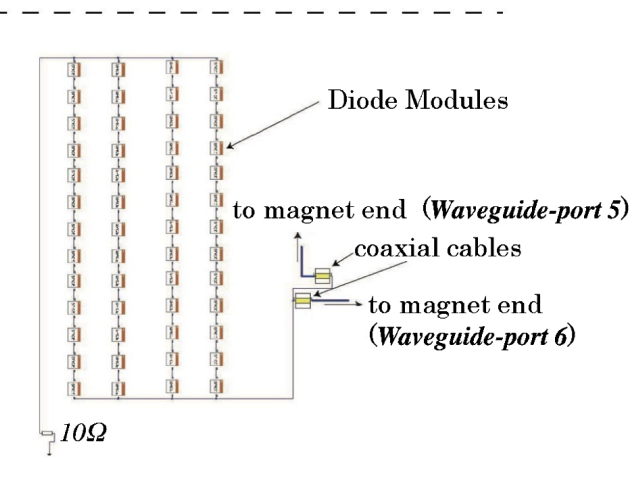

FIG. 11. Overall view of simulation setup for single wire measurements (upper left), the magnified figure of the kicker magnet part (lower left) and the terminal condition of all cables (right). 
Fourier-transformed current at terminal two; and $S_{c c(d d) 21}$ and $Z_{c c(d d)}$ denote the scattering matrix and the characteristic impedance for the common (differential) mode, respectively.

By substituting the calculated matrices into Eq. (4) or Eq. (5), we finally obtain the beam impedance of the kicker when connected to cables terminated by the diodes and resistors. The simulation results are shown in Fig. 12. The green open circle, black square, blue diamond, and red filled circle lines show the results for $1.245 \times 10^{13}$ (300 kW-eq), $2.075 \times 10^{13}(500 \mathrm{~kW}$-eq $), 3.11 \times 10^{13}$ $\left(750 \mathrm{~kW}\right.$-eq) and $4.15 \times 10^{13}$ (1 MW-eq) particles per bunch, respectively. This is why both the longitudinal and horizontal beam impedances of the kicker decrease, as the current in the wire (i.e., the beam intensity at the RCS) increases. In the next section, we investigate the intensitydependent reduction of the beam impedance using measurements with accelerated beams at the RCS.

\section{MEASUREMENT OF THE BEAM IMPEDANCE OF THE MODIFIED KICKER}

When a beam passes through the kicker, it excites currents in the $\mathrm{Al}$ coils in the kicker. Therefore, the beam impedance of the kicker can be derived by measuring the beam-induced voltages at the ends of the coaxial cables and the total currents of the two cables, and determining the effective impedance $R_{T}$ of the diodes and resistors.

Figure 13 shows one measurement of the beam-induced voltages at the ends of coaxial cables (left) and the total currents of two cables (right), when a beam with $3.11 \times 10^{13}$ particles per bunch $(750 \mathrm{~kW}$-eq $)$ passes through the kicker. The red, blue and green lines show the results for (i) open circuit, (ii) diodes and $10 \Omega$ resistors, and (iii) only $10 \Omega$ resistors, respectively. The horizontal axis denotes the acceleration time. When only the matched resistors are connected (green), the beam-induced voltages
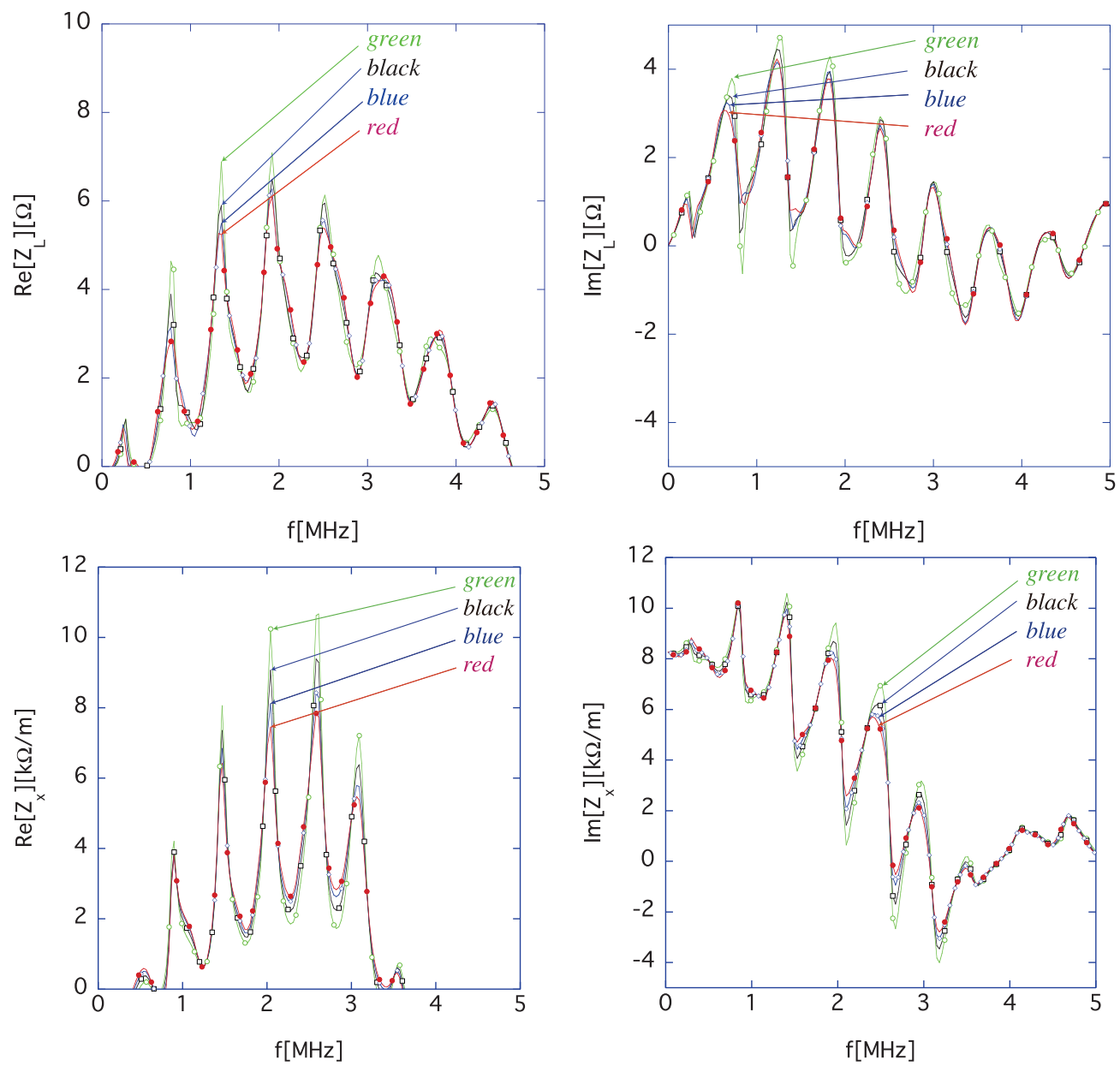

FIG. 12. Simulation results for the longitudinal (upper row) and horizontal (lower row) beam impedances, where the diodes and resistors are installed. The wire radius is $\rho_{w}=80 \mu \mathrm{m}$ and the twin-wire separation is $\Delta=60 \mathrm{~mm}$. The green open circle, black square, blue diamond, and red filled circle lines show the results for $1.245 \times 10^{13}\left(300 \mathrm{~kW}\right.$-eq), $2.075 \times 10^{13}\left(500 \mathrm{~kW}\right.$-eq), $3.11 \times 10^{13}$ $\left(750 \mathrm{~kW}\right.$-eq), and $4.15 \times 10^{13}(1 \mathrm{MW}$-eq) particles per bunch, respectively. The left and the right columns show the real and the imaginary parts of the beam impedances, respectively. 

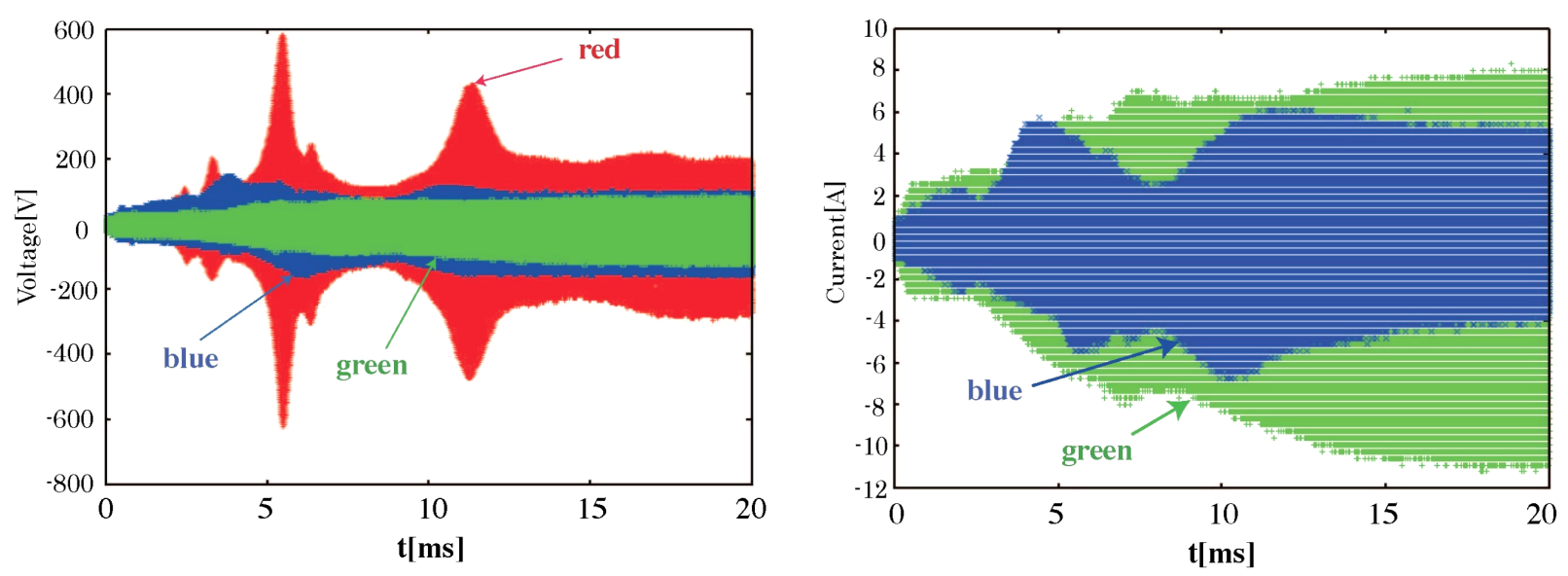

FIG. 13. Measurements of beam-induced voltages at the ends of coaxial cables (left) and the total currents of the two cables (right), when a beam with $3.11 \times 10^{13}(750 \mathrm{~kW}$-eq) particles per bunch passes through the kicker. The red, blue and green lines show the results for (i) open circuit, (ii) diodes and $10 \Omega$ resistors, and (iii) only $10 \Omega$ resistors, respectively.

are completely absorbed at the terminals. When the terminals are open (red), the reflection voltages are superposed, and they become maximum. When the diodes are inserted in front of the resistors (blue), the matching conditions are not perfectly satisfied. Consequently, the induced voltages (blue) lie between the case in which the terminals are open (red) and that in which the matched resistors are connected (green).

In the right part of Fig. 13, the beam-induced current increases for the case with only resistors connected (green) at the terminal ends, compared to that for the case with the diode additionally connected (blue). The beam-induced current at the terminal ends is increased, as the effective terminal impedance $R_{T}$ decreases. That is why the beaminduced current for the matched resistor (green) is higher than that for the diode with resistors (blue).

In order to clarify the beam-intensity dependence of the beam impedance from the measurements, let us measure the beam-induced voltages and currents for the cases with $1.245 \times 10^{13}\left(300 \mathrm{~kW}\right.$-eq), $2.075 \times 10^{13}(500 \mathrm{~kW}$-eq $)$ and $3.11 \times 10^{13}(750 \mathrm{~kW}$-eq) particles per bunch. Figure 14 shows the measurements of beam-induced voltages at the ends of coaxial cables (left) and the total currents of the two cables (right). The green, black, and blue lines show the results for $1.245 \times 10^{13}(300 \mathrm{~kW}$-eq $), 2.075 \times 10^{13}$ $\left(500 \mathrm{~kW}\right.$-eq), and $3.11 \times 10^{13}(750 \mathrm{~kW}$-eq) particles per bunch, respectively.

As the bottom figures of Table I illustrate, the V-I curve of diode rapidly rises, indicating an effective reduction in the diode's dynamic resistance. The blue line in the left figure of Fig. 14 indicates that the total voltage on the diode in Fig. 5 can be $150 \mathrm{~V}$ or higher, for the $750 \mathrm{~kW}$ or higher beam. Accordingly, the total dynamic resistance of the diode can be estimated as $3 \Omega$ or lower for the beam (refer to Sec. III). That is why $10 \Omega$ resistor is additionally connected to the anode of diode in Fig. 5.
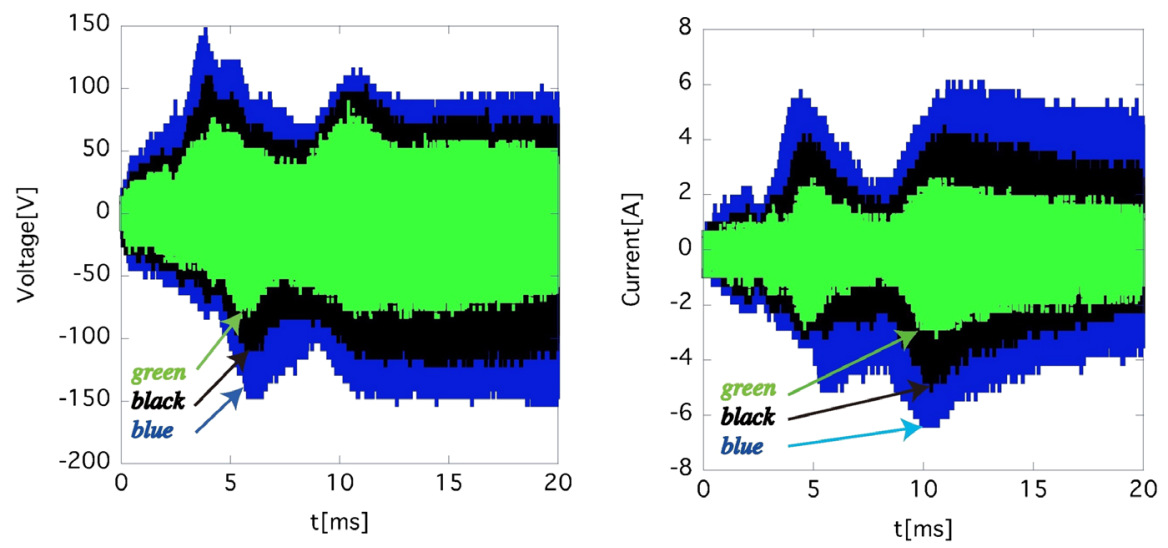

FIG. 14. Measurements of beam-induced voltages at the ends of coaxial cables (left) and the total currents of the two cables (right), when a beam passes through the kicker whose input cables are terminated with both diodes and resistors. The green, black and blue lines show the results for $1.245 \times 10^{13}\left(300 \mathrm{~kW}\right.$-eq), $2.075 \times 10^{13}\left(500 \mathrm{~kW}\right.$-eq), and $3.11 \times 10^{13}(750 \mathrm{~kW}$-eq) particles per bunch, respectively. 
As the beam-intensity increases, the beam-induced voltages (the left part of Fig. 14) increase, as expected. Moreover, the beam-induced currents (the right part of Fig. 14) increase more than the beam-induced voltages. Hence, we indirectly find that the beam impedances of the kicker system with the diodes and resistors are reduced more as the beam intensity increases, as the simulation results suggest in Fig. 12.

Fourier-transformed beam-induced voltage divided by Fourier-transformed beam-induced current is the effective impedance $R_{T}$ of the diode with resistors. By substituting the measured $R_{T}$ at Lorentz- $\beta=0.97$ into the formulas in the Appendix, we can obtain the beam impedance of the modified kicker for a relativistic beam. The results are shown in Fig. 15. The green open circle, black square and blue diamond lines show the results for $1.245 \times 10^{13}$ (300 kW-eq), $2.075 \times 10^{13}\left(500 \mathrm{~kW}\right.$-eq) and $3.11 \times 10^{13}$ (750 kW-eq) particles per bunch, respectively. The peaks of the beam impedance of the kicker are reduced by increasing the beam intensity, as expected.

Figure 16 summarizes the measurements (blue solid) and the simulation (blue dashed) results for $3.11 \times 10^{13}$
(750 kW-eq) particles per bunch when the cable terminals are terminated by the diodes with resistors, and the analytical (red) results when the cable terminals are open. The beam impedance for open terminal (red) is significantly reduced by terminating the ends of cables with the diodes and resistors (see the blue solid and blue dashed lines). The agreement between the blue solid and blue dashed lines is relatively good. However, we can still see a discrepancy between them, especially for the imaginary part of the horizontal beam impedance at low frequency. As already seen in Figs. 3 and 10, this limitation is unavoidable, due to the lack of the dispersion relation for the cable resistivity in the CST code. If we divide the total frequency range into narrower bins and assign effective attenuation parameters for each frequency bin, the discrepancy might be mitigated.

Apart from this discrepancy, the measurements demonstrate that the simulation technique developed in Sec. IV can provide a guideline to estimate the beam impedances of the kicker magnet connected to the 130-m-long coaxial cables terminated with the nonlinear devices (the diodes specified by the SPICE parameters) and resistors.
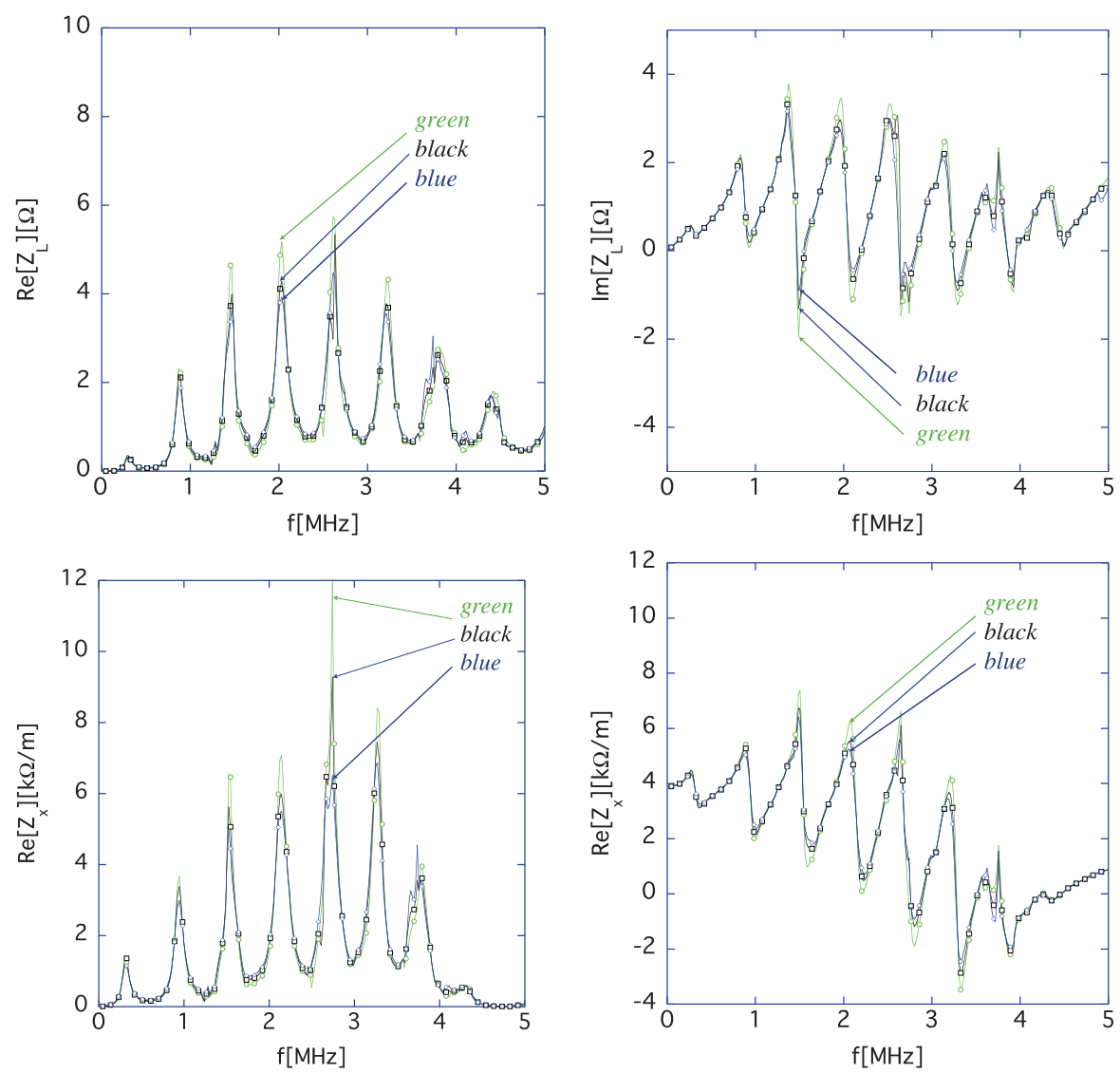

FIG. 15. Measurements of the longitudinal (upper row) and horizontal (lower row) beam impedances with beam-induced voltages and currents, where the diodes and resistors are installed. The green open circle, black square and blue diamond lines show the results for $1.245 \times 10^{13}\left(300 \mathrm{~kW}\right.$-eq), $2.075 \times 10^{13}\left(500 \mathrm{~kW}\right.$-eq), and $3.11 \times 10^{13}(750 \mathrm{~kW}$-eq) particles per bunch, respectively. The left and the right columns show the real and imaginary parts of the beam impedances, respectively. 

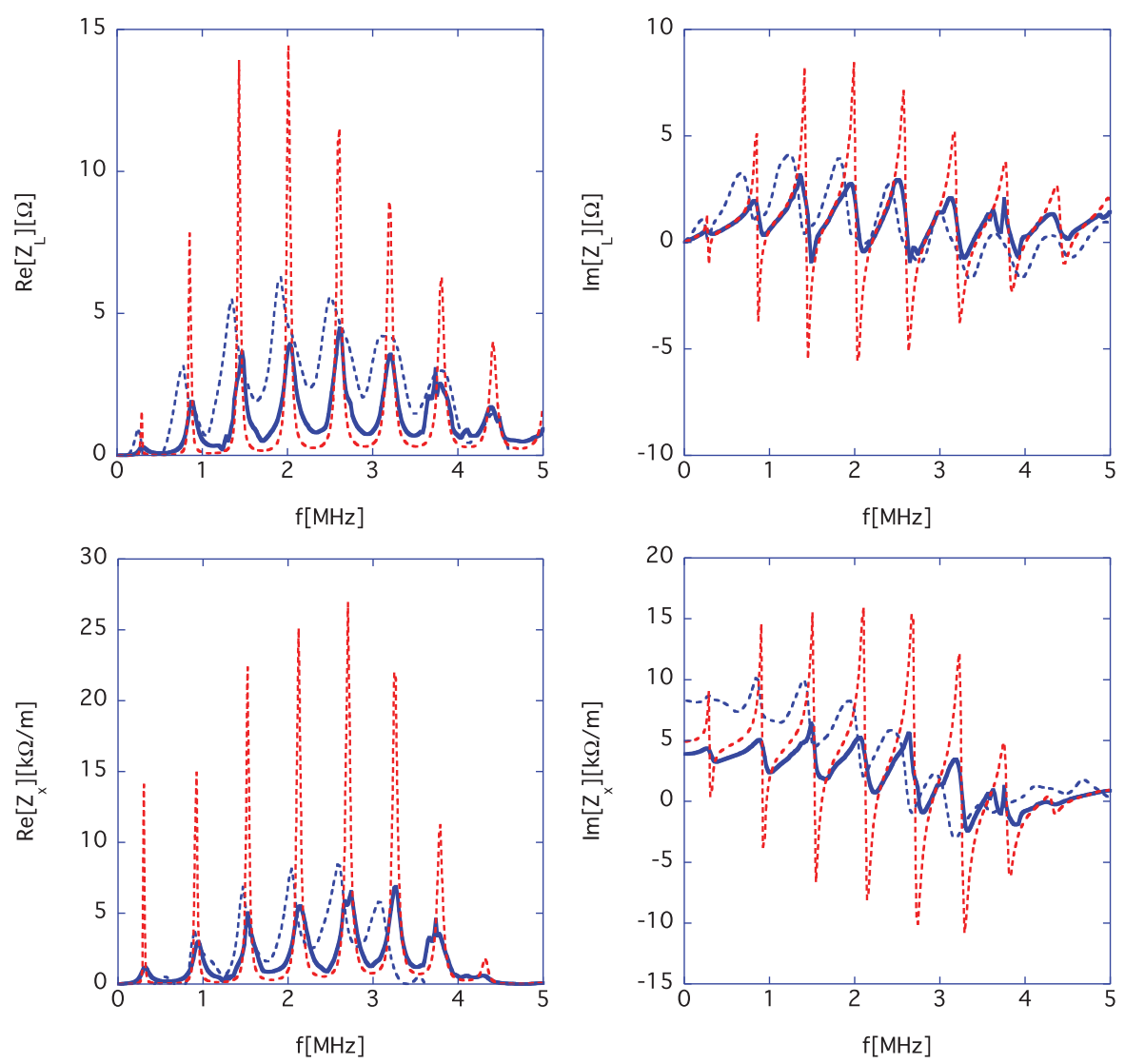

FIG. 16. The longitudinal (upper row) and horizontal (lower row) beam impedances for a relativistic beam. The blue solid and blue dashed lines show the measurements and the simulation results for $3.11 \times 10^{13}(750 \mathrm{~kW}$-eq) particles per bunch when the cable terminals are terminated by the diodes with resistors, respectively. The red line shows the analytical results when the cable terminals are open. The left and the right columns show the real and imaginary parts of the beam impedances, respectively.

\section{SUMMARY}

We have developed a scheme to reduce the beam impedance of the kicker at the RCS at J-PARC. The RCS kicker has four terminals, two of which are connected to 130-m-long coaxial cables, while the other two are shorted.

We have inserted diodes and resistors at the switch end of the coaxial cables. This ensures doubled excitation currents for beam extraction by inverting the voltage wave front and superposing the excitation currents with two shorted ends. In this scheme the resistors are isolated from the switch cathode, while they can be seen by high intensity beam-induced currents.

Intuitively, this scheme can reduce the beam impedance of the kicker, but there has been no way to determine the beam impedance from either simulations or measurements. The standard measurement scheme, using a network analyzer in the frequency domain, is useless, because nonlinear devices, i.e., diodes, are present in the kicker system. Since the precision of an oscilloscope is worse than that of a network analyzer, by an order of magnitude, it is quite difficult to determine the impedances precisely by typical measurements in the time domain. Moreover, in order to observe the dependence of the working diode on the beam intensity, a high peak current is indispensable.
However, we have now succeeded in measuring the beam impedance of the kicker by taking advantage of a high-intensity beam at the RCS. By measuring the beaminduced voltages and currents at the diodes with resistors, which are connected to the ends of the coaxial cables, we can determine the beam impedance of the kicker by substituting the measured effective impedance of the diodes and resistors into a previously obtained formula.

From the simulation point of view, since 130-m-long cables are connected to the kicker, it is almost impossible to carry out a three-dimensional simulation, because excessive memory and CPU time are necessary. We have overcome this obstacle by means of a simulation of the measurement setup, stretching wires inside the kicker, and making use of the MicroWave Solver, bypassing the WaKe Solver, of the code CST STUdio SuITE 2017. One waveguide port is connected to a pulse generator and the other to the ground through a matched resistor. The other waveguide ports are connected to the 130-m-long cables terminated by the resistors and diodes designated by SPICE parameters at the electric circuit part of CST code. Finally, we have calculated the dependence of the beam impedance of the kicker on the current intensity using this simulation technique. 
The measurement and the simulation results are in reasonably good agreement. Consequently, we conclude that we have successfully determined the beam impedance of the kicker quantitatively, and this scheme is beneficial for reducing the beam impedance while retaining the function of beam extraction from the RCS.

\section{ACKNOWLEDGMENTS}

The authors would like to thank all members of the JPARC project at JAEA/KEK. They also would like to thank all members of the J-PARC Accelerator Technical Advisory Committee, which was led by Dr. Steve Holmes until 2009, and has been led by Dr. Thomas Roser from 2010 until 2018.

\section{APPENDIX: THE KICKER IMPEDANCES WITH COAXIAL CABLES, THE ENDS \\ OF WHICH ARE TERMINATED BY THE IMPEDANCE $R_{T}$}

The longitudinal impedance $Z_{L}$ and the horizontal impedance $Z_{x}$ are derived in Ref. [8]. The formulas for a relativistic beam can be summarized as

$$
\begin{aligned}
& Z_{L}=Z_{L}^{(0)}+Z_{L}^{(\mathrm{crr})}, \\
& Z_{x}=Z_{x}^{(1)}+Z_{x}^{(\mathrm{crr})},
\end{aligned}
$$

where

$$
\begin{aligned}
& Z_{L}^{(0)}=2 \sum_{m=1}^{\infty} \frac{\left[-(-1)^{m}+1+\cos \frac{m \pi\left(\rho_{p}+a\right)}{2 a}-\cos \frac{m \pi\left(-\rho_{p}+a\right)}{2 a}\right] \sin \left(\frac{m \pi}{2}\right)}{m \pi} \\
& \times\left[\frac{c \theta_{1} Z_{c, c}\left(e^{j\left(k+\omega \theta_{1}\right) L}-1\right) F_{3}}{q\left(1+c \theta_{1}\right) \cosh \sqrt{k_{x m}^{\prime 2}+\omega^{2} \theta_{1}^{2}-k^{2}} b}+\frac{c \theta_{1} Z_{c, c}\left(e^{j\left(k-\omega \theta_{1}\right) L}-1\right) F_{4}}{q\left(1-c \theta_{1}\right) \cosh \sqrt{k_{x m}^{\prime 2}+\omega^{2} \theta_{1}^{2}-k^{2} b}}+\frac{j \omega L M_{+}\left(x_{0}=0\right)}{\cosh \frac{m \pi}{2 a} b}\right], \\
& Z_{L}^{(\mathrm{crr})}=-\frac{L_{f}}{a} \sum_{m=1}^{\infty} E_{m}^{\gamma \rightarrow \infty} \sin \left(\frac{m \pi}{2}\right) \\
& Z_{x}^{(1)}=-\left.\frac{c \theta_{3} Z_{c, d}\left(e^{j\left(k+\omega \theta_{3}\right) L}-1\right)}{q a\left(k+\omega \theta_{3}\right)} \frac{d F_{1}}{d x_{0}}\right|_{x_{0}=0}-\left.\frac{c \theta_{3} Z_{c, d}\left(e^{j\left(k-\omega \theta_{3}\right) L}-1\right)}{q a\left(k-\omega \theta_{3}\right)} \frac{d F_{2}}{d x_{0}}\right|_{x_{0}=0}+\left.\frac{j c L}{a} \frac{d M_{-}}{d x_{0}}\right|_{x_{0}=0}, \\
& Z_{x}^{(\mathrm{crr})}=-\left.\frac{L_{f}}{k a} \sum_{m=1}^{\infty} \frac{d E_{m}^{\gamma \rightarrow \infty}}{d x_{0}}\right|_{x_{0}=0} k_{x m}^{\prime} \cos k_{x m}^{\prime} a, \\
& E_{m}^{\gamma \rightarrow \infty}=-j k Z_{0}\left(-1+\mu^{\prime} \epsilon^{\prime}\right) \frac{\left[\frac{2 C_{r}\left(x_{0}=0\right) \cosh \left(k_{x m}^{\prime} b\right)\left\{\cosh \left[k_{y m}(b-B)\right]-1\right\} \sin \frac{m \pi}{2}}{k_{y m} \sinh \left[k_{y m}(b-B)\right]}-\sin \left(k_{x m}^{\prime} a\right)\right] \frac{1}{\sinh 2 k_{x m}^{\prime} b}}{k_{y m} \frac{2\left\{\epsilon^{\prime} \cosh ^{2}\left(k_{x m}^{\prime} b\right) \operatorname{coth}\left[k_{y m}(b-B)\right]+\mu^{\prime} \sinh ^{2}\left(k_{x m}^{\prime} b\right) \tanh \left[k_{y m}(b-B)\right]\right\}}{\sinh 2 k_{x m}^{\prime} b}-k_{x m}^{\prime}\left(1+\epsilon^{\prime} \mu^{\prime}\right)}, \\
& \left.\frac{d E_{m}^{\gamma \rightarrow \infty}}{d x_{0}}\right|_{x_{0}=0}=\frac{j k Z_{0}\left(-1+\mu^{\prime} \epsilon^{\prime}\right)}{a} \frac{\frac{k_{x m}^{\prime} \cos k_{x m}^{\prime} a}{\sinh 2 k_{x m}^{\prime} b}}{k_{y m} \frac{2\left\{\epsilon^{\prime} \cosh ^{2}\left(k_{x m}^{\prime} b\right) \operatorname{coth}\left[k_{y m}(b-B)\right]+\mu^{\prime} \sinh ^{2}\left(k_{x m}^{\prime} b\right) \tanh \left[k_{y m}(b-B)\right]\right\}}{\sinh 2 k_{x m}^{\prime} b}-k_{x m}^{\prime}\left(1+\epsilon^{\prime} \mu^{\prime}\right)},
\end{aligned}
$$

$$
\begin{aligned}
C_{r}\left(x_{0}\right) & =-\frac{1}{2(B-b)}\left(1-\frac{M^{r}\left(x_{0}\right)+M^{l}\left(x_{0}\right)}{L_{k}+M_{b}}\right), \\
\theta_{1} & =\sqrt{\left(L_{k}+M_{b}\right) C_{k}}, \\
\theta_{3} & =\sqrt{\left(L_{k}-M_{b}\right) C_{k}}, \\
Z_{c, d} & =\sqrt{\frac{L_{k}-M_{b}}{C_{k}}},
\end{aligned}
$$

$$
\begin{aligned}
& Z_{c, c}=\sqrt{\frac{L_{k}+M_{b}}{C_{k}}}, \\
& k_{x m}^{\prime}=\frac{m \pi}{2 a}, \\
& k_{y m}=\sqrt{k_{x m}^{\prime 2}+k^{2}\left(1-\epsilon^{\prime} \mu^{\prime}\right)} .
\end{aligned}
$$

Here $\omega=2 \pi f$ is the angular frequency, $k=\omega / c, c$ is the velocity of light, $\epsilon^{\prime}$ and $\mu^{\prime}$ are the relative dielectric constant and the relative permeability of the ferrite, respectively, 
$Z_{0}=120 \pi \Omega$ is the impedance of free space, $L$ is the total length of the kicker, $L_{f}$ is the total length of the ferrite component of the kicker, $2 a$ and $2 b$ are the gap width and gap height, respectively, $2 \rho_{p}$ is the distance between the high voltage plates as shown in Fig. 2, the parameter $C_{k}(=13.62) \mathrm{nF} / \mathrm{m}$ is the capacitance of the kicker per unit length, $q$ is the charge of a bunch in the beam, $x_{0}$ is the horizontal position of the beam, and $B-b$ is the thickness of the ferrite (see Fig. 2).

The parameters $L_{k}$ and $M_{b}$ are the self-inductance of the kicker per unit length and the mutual inductance between the coils per unit length, respectively, which are calculated as

$$
\begin{aligned}
L_{k}= & -\frac{\mu_{0}}{4 b}\left[-\frac{K_{1}\left(x_{1}\right)}{\sin \left(\frac{2 \omega a}{c}\right)}+\frac{K_{2}}{\sin \left(\frac{\omega a}{c}\right)}+\sum_{m=1}^{\infty} \frac{\left[1+(-1)^{m}\right] K_{3}}{\sinh \left(\theta_{m 0} a\right)}\right], \\
M_{b}= & -\frac{\mu_{0}}{4 b}\left[-\frac{K_{1}\left(x_{2}\right)}{\sin \left(\frac{2 \omega a}{c}\right)}+\frac{K_{2}}{\sin \left(\frac{\omega a}{c}\right)}+\sum_{m=1}^{\infty} \frac{\left[1+(-1)^{m}\right] K_{3}}{\sinh \left(\theta_{m 0} a\right)}\right], \\
K_{1}\left(x_{i}\right)= & -\frac{c}{\omega}\left[\cos \frac{\omega\left(a+x_{i}\right)}{c}-\cos \frac{\omega\left(\rho_{w}+x_{i}\right)}{c}\right] \\
& -\frac{c}{\omega}\left[\cos \frac{\omega\left(x_{i}+a\right)}{c}-\cos \frac{\omega\left(\left|x_{i}-\rho_{w}\right|-2 a\right)}{c}\right], \\
K_{2}= & -\frac{c}{\omega}\left[1-\cos \frac{\omega\left(\rho_{w}-a\right)}{c}\right], \\
K_{3}= & \frac{1-\cosh \left[\theta_{m 0}\left(\rho_{w}-a\right)\right]}{\theta_{m 0}}, \\
K_{4}\left(x_{0}\right)= & \frac{\cosh \left[\theta_{m 0}\left(a+x_{0}\right)\right]-\cosh \left[\theta_{m 0}\left(\rho_{w}+x_{0}\right)\right]}{\theta_{m 0}} \\
& +\frac{\cosh \left[\theta_{m 0}\left(x_{0}+a\right)\right]-\cosh \left[\theta_{m 0}\left(\left|x_{0}-\rho_{w}\right|-2 a\right)\right]}{\theta_{m 0}},
\end{aligned}
$$

where

$$
\begin{aligned}
\mu_{0} & =\frac{Z_{0}}{c}, \\
\theta_{m 0} & =\sqrt{\frac{m^{2} \pi^{2}}{4 b^{2}}-\frac{\omega^{2}}{c^{2}}}, \\
x_{1} & =a, \\
x_{2} & =-a,
\end{aligned}
$$

and $\rho_{w}$ is the half-width of the flux break (see Fig. 2).
The parameters $M^{r}\left(x_{0}\right)$ and $M^{l}\left(x_{0}\right)$ are the induction coefficients between the beam and the right coil and between the beam and the left coil, respectively, which are calculated as

$$
\begin{aligned}
M^{r}\left(x_{0}\right) & =-\frac{\mu_{0}}{4 b}\left\{-\frac{K_{1}\left(x_{0}\right)}{\sin \left(\frac{2 \omega a}{c}\right)}+\frac{K_{2}}{\sin \left(\frac{\omega a}{c}\right)}\right. \\
& \left.+\sum_{m=1}^{\infty}\left[-\frac{2(-1)^{m} \cos \left(\frac{m \pi}{2}\right) K_{4}\left(x_{0}\right)}{\operatorname{sh}\left(2 \theta_{m 0} a\right)}+\frac{\left[1+(-1)^{m}\right] K_{3}}{\sinh \left(\theta_{m 0} a\right)}\right]\right\},
\end{aligned}
$$

$M^{l}\left(x_{0}\right)=M^{r}\left(-x_{0}\right)$,

$M_{-}\left(x_{0}\right)=\frac{\left[M^{r}\left(x_{0}\right)-M^{l}\left(x_{0}\right)\right]}{2\left[1-c^{2}\left(L_{k}-M_{b}\right) C_{k}\right]}$,

$M_{+}\left(x_{0}\right)=\frac{\left[M^{r}\left(x_{0}\right)+M^{l}\left(x_{0}\right)\right]}{2\left[1-c^{2}\left(L_{k}+M_{b}\right) C_{k}\right]}$.

The functions $F_{1}(\omega), F_{2}(\omega), F_{3}(\omega)$ and $F_{4}(\omega)$ are determined by solving

$$
\begin{aligned}
& \left(Z_{c, d}-\mathcal{Z}_{\text {cable }}\right) F_{1}-\left(Z_{c, d}+\mathcal{Z}_{\text {cable }}\right) F_{2}-\left(Z_{c, c}-\mathcal{Z}_{\text {cable }}\right) F_{3} \\
& \quad+\left(Z_{c, c}+\mathcal{Z}_{\text {cable }}\right) F_{4} \\
& =-\left(M_{-}+M_{+}\right) q c-\mathcal{Z}_{\text {cable }} q C_{k} c^{2}\left(M_{-}+M_{+}\right), \\
& -Z_{c, d} F_{1}+Z_{c, d} F_{2}-Z_{c, c} F_{3}+Z_{c, c} F_{4}=q c\left(M_{-}-M_{+}\right),
\end{aligned}
$$

$$
\begin{aligned}
& Z_{c, d} e^{j \omega \theta_{3} L} F_{1}-Z_{c, d} e^{-j \omega \theta_{3} L} F_{2}-Z_{c, c} e^{j \omega \theta_{1} L} F_{3} \\
& \quad+Z_{c, c} e^{-j \omega \theta_{1} L} F_{4}=-\left(M_{-}+M_{+}\right) q c e^{-j \omega \frac{L}{c}}, \quad(\mathrm{~A} 32) \\
& -\left(Z_{c, d}+\mathcal{Z}_{\text {cable }}\right) e^{j \omega \theta_{3} L} F_{1}+\left(Z_{c, d}-\mathcal{Z}_{\text {cable }}\right) e^{-j \omega \theta_{3} L} F_{2} \\
& \quad-\left(Z_{c, c}+\mathcal{Z}_{\text {cable }}\right) e^{j \omega \theta_{1} L} F_{3}+\left(Z_{c, c}-\mathcal{Z}_{\text {cable }}\right) e^{-j \omega \theta_{1} L} F_{4} \\
& =\left(M_{-}-M_{+}\right) q c e^{-j \omega \frac{L}{c}}-\mathcal{Z}_{\text {cable }}\left(M_{-}-M_{+}\right) q C_{k} c^{2} e^{-j \omega \frac{L}{c}},
\end{aligned}
$$

which describes the boundary conditions (two ends are terminated in a short circuit, while the other two are connected to the coaxial cables), where

$$
\mathcal{Z}_{\text {cable }}=\frac{1}{2} \sqrt{\frac{L_{\text {cable }}}{C_{\text {cable }}}} \frac{\left(1+\frac{-\sqrt{\frac{L_{\text {cable }}}{C_{\text {cable }}}}+2 R_{T}}{\sqrt{\frac{L_{\text {cable }}}{C_{\text {cable }}}+2 R_{T}}} e^{-j \omega 2 \sqrt{C_{\text {cable }} L_{\text {cable }}} l_{\text {cable }}}\right)}{\left(1+\frac{-\sqrt{\frac{L_{\text {cable }}}{C_{\text {cable }}}}+2 R_{T}}{-\sqrt{\frac{L_{\text {cable }}}{C_{\text {cable }}}-2 R_{T}}} e^{-j \omega 2 \sqrt{C_{\text {cable }} L_{\text {cable }}} l_{\text {cable }}}\right)},
$$




$$
\begin{aligned}
L_{\text {cable }} & =\mathcal{L}_{\text {cable }}+\frac{R_{\text {cable }}}{j \omega}, \\
C_{\text {cable }} & =\mathcal{C}_{\text {cable }}+\frac{G}{j \omega} .
\end{aligned}
$$

The quantities $\mathcal{L}_{\text {cable }}$ and $\mathcal{C}_{\text {cable }}$ are the inductance per unit length and the capacitance per unit length of the cable, the resistivity $R_{\text {cable }}$ and the conductance $G$ are given by Eqs. (1) and (2), respectively. Note that Eq. (A34) is a bit different from Eq. (D.11) in Ref. [8], because it deals with the case that two parallel cables are connected at the ends and terminated with one electric device having the impedance $R_{T}(\omega)$ [3]. The results for an open circuit, shown in Figs. 3 and 16, are obtained by substituting infinity for the terminal impedance $R_{T}$.

When diodes with resistors are connected to the ends of the coaxial cables, the beam-induced voltages at the ends of coaxial cables and the total currents of two cables are determined by letting a beam pass through the kicker. Accordingly, the effective terminal impedance $R_{T}$ can be measured for the given beam. By substituting the value of $R_{T}$ into Eq. (A34), we obtain the kicker impedances shown in Figs. 15 and 16.

[1] http://j-parc.jp/index-e.html.

[2] A. W. Chao, Physics of Collective Beam Instabilities in High Energy Accelerators (Wiley, New York, 1993).

[3] Y. Shobuda, P. K. Saha, T. Toyama, M. Yamamoto, Y. H. Chin, and Y. Irie, The kicker impedance and its effect on the RCS in J-PARC, in Proceedings of HB2014, Michigan, USA, 2014, p. 369, http://epaper.kek.jp/HB2014/papers/ tho2ab02.pdf.

[4] Y. Shobuda, N. Hayashi, T. Takayanagi, Y. Toyama, and Y. Irie, A trial to reduce the kicker impedance of 3-GeV RCS in J-PARC, in Proceedings of the 4th International Particle Accelerator Conference, IPAC-2013, Shanghai, China, 2013, p. 1742, http://accelconf.web.cern.ch/accelconf/ IPAC2013/papers/tupwa010.pdf.

[5] Y. Shobuda, Y. H. Chin, P. K. Saha, H. Hotchi, H. Harada, Y. Irie, F Tamura, N. Tani, T. Toyama, Y. Watanabe, and M. Yamamoto, Theoretical elucidation of space-charge effects on the coupled-bunch instability at the $3 \mathrm{GeV}$ rapid cycling synchrotron at the Japan Proton Accelerator Research Complex, Prog. Theor. Exp. Phys. (2017) 013G01.
[6] P. K. Saha, Y. Shobuda, H. Hotchi, H. Harada, N. Hayashi, M. Kinsho, F. Tamura, N. Tani, M. Yamamoto, Y. Watanabe, Y. H. Chin, and J. A. Holmes, Simulation, measurement, and mitigation of beam instability caused by the kicker impedance in the 3-GeV rapid cycling synchrotron at the Japan Proton Accelerator Research Complex, Phys. Rev. Accel. Beams 21, 024203 (2018).

[7] J. Kamiya, T. Takayanagi, and M. Watanabe, Performance of extraction kicker magnet in a rapid cycling synchrotron, Phys. Rev. ST Accel. Beams 12, 072401 (2009).

[8] Y. Shobuda, Y. Irie, T. Toyama, J. Kamiya, and M. Watanabe, Measurement scheme of kicker impedances via beam-induced voltages of coaxial cables, Nucl. Instrum. Methods Phys. Res., Sect. A 713, 52 (2013).

[9] J. Kamiya, T. Ueno, and T. Takayanagi, Magnetic field measurement of the extraction kicker magnet in J-PARC RCS, IEEE Trans. Appl. Supercond. 16, 1362 (2006).

[10] http://www.global.tdk.com/corp/en/index.htm.

[11] Y. Shobuda, Y. Irie, and T. Toyama, Analytical approach to evaluate coupling impedances of traveling kicker magnets, Nucl. Instrum. Methods Phys. Res., Sect. A 691, 135 (2012).

[12] Y. Shobuda, Y. H. Chin, and K. Takata, Impedance of a ceramic break and its resonance structures, Phys. Rev. ST Accel. Beams 17, 091001 (2014).

[13] Handbook of Accelerator Physics and Engineering, edited by A. W. Chao and M. Tigner (World Scientific, Singapore, 1999).

[14] H. J. Tran, M. J. Barnes, G. D. Wait, and Y. Yan, Longitudinal impedance of a prototype kicker magnet system, in Proceedings of the 15th Particle Accelerator Conference, PAC-1993, Washington, DC, 1993 (IEEE, New York, 1993), p. 3502, https://ieeexplore.ieee.org/ document/309664/.

[15] G. Nassibian and F. Sacherer, A method for measuring transverse coupling impedance, Nucl. Instrum. Methods 159, 21 (1979).

[16] https://www.origin.co.jp/eng/.

[17] Y. Shobuda, Y. Irie, T. Takayanagi, T. Togashi, K. Yamamoto, and M. Yamamoto, Reduction of the kicker impedance maintaining the performance of present kicker magnet at RCS in J-PARC, in Proceedings of IPAC 2018, Vancouver, Canada, 2018 (to be published).

[18] CST Studio Suite, https://www.cst.com.

[19] Y. Shobuda and Y. H. Chin, Resistive-wall impedances of a thin neg coating on a conductive chamber, Prog. Theor. Exp. Phys. 2017, 123G01 (2017).

[20] For example, C. A. Paul, Introduction to Electromagnetic Compatibility, 2nd ed. (John Wiley Interscience, New York, 2006). 